\title{
Toward a Local Theory of Light
}

\author{
Richard A. Hutchin \\ Optical Physics Company, Calabasas, CA, USA \\ Email: rahutchin@opci.com
}

Received 14 May 2015; accepted 28 July 2015; published 31 July 2015

Copyright (C) 2015 by author and Scientific Research Publishing Inc.

This work is licensed under the Creative Commons Attribution International License (CC BY).

http://creativecommons.org/licenses/by/4.0/

CC) (7) Open Access

\section{Abstract}

Quantum mechanics is a probabilistic theory of the universe suggestive of a mean value theory similar to thermodynamics prior to the introduction of the atomic theory. If QM will follow a similar path to thermodynamics, then a local deterministic theory must be developed which matches QM predictions. There have been four tough barriers to a local theory of light, of which Bell's Theorem has been considered the ultimate barrier. The other three barriers are explaining spontaneous emission, the reflection of a small fraction of light at a dielectric interface and the splitting action of a polarizer on polarized light (Malus' Law). The challenge is that in a local theory of light, everything must have a specific cause and effect. There can be nothing spontaneous or hidden. Local solutions to all four of these barriers are presented in this paper, integrating results from two previous papers and adding the solution paths to the third and fourth barriers as well, which are nearly identical. A previous paper [1] used results from Einstein's famous 1917 paper on stimulated emission to provide a deterministic local model for spontaneous emission. A second paper [2] showed that QM predictions in tests of Bell's theorem could be matched with a local model by modifying the definition of entanglement in a manner invisible to quantum mechanics. This paper summarizes and extends those two results and then presents a deterministic model of reflection from a dielectric interface and transmission of polarized light through a polarizer that both match quantum mechanics. As the framework of a local theory of light emerges, it is not surprising that we find corners of physics where small disagreements with quantum mechanics are predicted. A new Bell type test is described in this paper which can distinguish the local from the nonlocal theory, giving predictions that must disagree slightly but significantly with quantum mechanics. If such experiments are proven to disagree with quantum mechanics, then the door to a local theory of light will be opened.

\section{Keywords}

Entangled Photons, Bell's Theorem, Local Theory of Light, Tests of Quantum Mechanics, Spontaneous Emission, Malus' Law, Reflection from a Dielectric 


\section{Introduction}

Quantum mechanics has several key issues that remain unresolved after a hundred years of research. Consider the collapse of a probability wave. What exactly is a probability wave and what makes it collapse? Even more consider the complexity. There are an estimated $10^{78}-10^{82}$ protons in the universe, first estimated by Eddington [3], and we have estimated that the cumulative emission of all stars in the universe have launched over $10^{90}$ photons into space, most of which are still sailing through the cosmos. According to quantum mechanics, all those photons do not really exist anywhere or perhaps exist at all possible places until the moment they collapse to excite some electron or scatter off a particle. Given that most of those photons have been traveling for over a billion light years, a usual dipole emission probability would have them anywhere in $10^{18}$ square light years, which includes about $10^{62}$ possible positions (assuming each position is $1 \times 1 \mathrm{um}$ in size). Since almost all those photons are currently traveling through the cosmos, somehow the universe must keep track of $10^{62} \times 10^{90}=10^{152}$ positions of current photons updating them all every 1 um or so of propagation (every $3 \times 10^{-15} \mathrm{sec}$ ) and communicating among all those positions so that when a photon does finally appear somewhere, the universe knows which one it is and turns off its probability wave instantaneously at all other $10^{62}$ possible places that one photon may have been. As a side note, this actually requires communicating back in time for all coordinate systems since any place above the past light cone is "simultaneous" in some Lorentz frame.

A very complicated universe indeed is required by quantum mechanics, which must someday address the question of how all that communication and tracking is accomplished.

Alternatively a local theory says that each photon is always in a single location, not somewhere in $10^{62}$ locations. Furthermore the photon would follow simple local laws on its propagation and interactions. Such a theory would be $10^{62}$ times less complicated than QM and would not require a hidden faster than light communication system. This level of simplification of $10^{62}$ is like replacing 100,000 solar systems with a single proton. That is the motivation to find a local theory of light.

The structure of this paper is summarized here:

1) Overview of a prior paper [1] about the vacuum field, leading to a local and deterministic model of stimulated emission.

2) A general discussion of several photonic clues from historical research.

3) The concept of photons being carried by the E \& M River is presented with a covariant equation for photonic flux.

4) A local theory of light is developed for how each photon decides to reflect or not at a dielectric transition, such as a glass surface.

5) Application of the local theory of dielectric reflection to the problem of polarized light partially reflecting and partially transmitting through a polarizer (Malus’ Law).

6) An overview and extension of a prior paper [2] on Bell's test for entangled photons, which provides a local solution to entangled light that matches QM predictions.

7) A new test is derived for a local model of light from the work on Bell's Test for entangled photons that will measurably disagree with quantum mechanics under conditions of high quantum efficiency.

We respectfully caution the reader that exploring a relatively unknown area of science, such as a local theory of light, inevitably leads to qualitative and as well as quantitative logic. We know from Bell's theorem that a local theory of light will be very different from classical E \& M theory, so one can expect new paradigm's anchored in experiment and theory but also extrapolating to deeper levels not yet well explored. As always, experiments will get the last word.

\section{A New Physical Model for the Vacuum Field Based on Einstein's Stimulated Emission Theory [1] Overview}

We can get insights into the photon by addressing the mystery of the vacuum field, sometimes called the zeropoint background. The vacuum E \& M field comes from the $1 / 2 h v$ energy in the lowest state of a quantum oscillator, and it has the power spectral field density $I_{v a c}$ of Equation (1) when integrated over all directions (i.e. $4 \pi$ steradians) [4].

$$
I_{v a c}(v)=\frac{4 \pi h c}{\lambda^{3}}=\frac{4 \pi h v^{3}}{c^{2}}\left(\text { watt } / \mathrm{cm}^{2} / \mathrm{Hz}\right)
$$


It was first proposed to be real and experimentally observable by Hedrick Casimir, a Dutch scientist, in 1948 [5]. Subsequently, many experiments have confirmed that it has the expected field strength, which is measured by the pressure exerted on two closely spaced reflecting surfaces [6]. For radiation longer than $10 \mathrm{~nm}$ wavelength, this vacuum field density is about $1.8 \times 10^{12}$ watt $/ \mathrm{cm}^{2}$ - a massive intensity equal to the entire power generation of our world in $10 \mathrm{~cm}^{2}$. As a result of this huge electromagnetic intensity, the force from the pressure of light on two parallel plates a distance d apart is simply given by Equation (18), which computes to 18.85 psi for a separation $d=10$ nanometers. The minus sign means that the force is squeezing the plates together.

$$
P_{v a c}=-\frac{\hbar c \pi^{2}}{240 d^{4}}
$$

Ref [6] summarizes past research in over 200 pages, validating the Casimir force well.

The biggest mystery of the vacuum field is that it has the E \& M field strength of 2 terawatt $/ \mathrm{cm}^{2}$ but does not burn up everything as a normal E \& M field of this strength would easily do. The conclusion is that the fields are there as predicted (because we measure their pressure), but they must have something different about them that prevents them from vaporizing everything.

The hint of this mysterious property we find in Clue 1, from Einstein's paper on stimulated emission [7].

Clue 1: The spontaneous emission coefficient $A_{21}$ has a simple physical relationship to the stimulated emission coefficient $B_{21}$ given by:

$$
A_{21}=\frac{B_{21} 8 \pi h v^{3}}{c^{2}}=\frac{1}{\tau_{\text {decay }}}
$$

In words, this result from Einstein's paper says:

The spontaneous decay of any atom is identical to stimulated emission from a background spectral intensity equal to twice the vacuum background $\left(4 \pi h v^{3} / c^{2}\right.$ in Equation (1)).

What is the meaning of Einstein's Equation (3), which has been mostly ignored? The logic we propose is the following:

Hypothesis 1: Light comes in two independent and separable forms, up stimulating and down stimulating, with the vacuum form being purely down stimulating.

This means that the vacuum field will have the field strength that is observed, but it has an additional quality that makes it purely down stimulating and is thus unable to burn everything up. To explore what quality would make an E \& M field down stimulating only, we use another result from the same Einstein paper:

Clue 2: Blackbody light has, on average, an equal ability to cause an up transition (excitation) as it does to cause a down transition (stimulated emission). Mathematically Einstein expressed this relationship as $B_{12}=B_{21}$, which means the cross-section for going from ground state 1 to excited state 2 equals the crosssection for stimulating the transition from excited state 2 to excited state 1.

Focusing on Clue 2, we ask how can the real fields of blackbody emission be divided into two equal components-one causing excitation and one causing stimulated emission? Our suggestion is to decompose all radiation into $\mathrm{e}^{+i w t}$ and $\mathrm{e}^{-i w t}$ components, where $\mathrm{e}^{+i w t}$ is up stimulating (excitation) and $\mathrm{e}^{-i w t}$ is down stimulating (stimulated emission). The sign convention is obviously arbitrary. When we look at power (defined here as mean magnitude-squared since we are dealing with complex fields), we get that half the power of a real field goes into the up-stimulating waveform and half into the down — an equal amount to each as required from Einstein's paper with $B_{12}=B_{21}$.

For instance, a real $\mathrm{E} \& \mathrm{M}$ field of the form $\cos (k z-c t)$ will decompose into up and down stimulating terms as shown in Equation (4). The mean-square-magnitude of the left side is $1 / 2$, while the mean-square-magnitude of each of the right terms is $1 / 4$. Note that the first term is purely $\mathrm{e}^{-i w t}$ and the second purely $\mathrm{e}^{+i w t}$.

$$
\cos (k z-\omega t)=\frac{\mathrm{e}^{i(k z-\omega t)}}{2}+\frac{\mathrm{e}^{-i(k z-\omega t)}}{2}
$$

With this half and half power division into up and down stimulating fields, we modify Einstein's result so that the up-stimulating capability equals $2 B_{12}$ times the power in the up stimulating component, and the down stimulating capability equals $2 B_{21}$ times the power in the down stimulating component. Since half the power of the real field goes into each up and down component, the final product is the same as he presented, only the inter- 
pretation has changed.

Applying this result to the vacuum field, which we model as purely down stimulating, we get that the ability of the vacuum field to stimulate emission is $2 B_{21} 4 \pi h c / \lambda^{3}=B_{21} 8 \pi h c / \lambda^{3}$ exactly as Einstein specified in Equation (3).

Now we summarize this in a more complete set of hypotheses:

Hypothesis 1: Light comes in two independent and separable forms, $\mathrm{e}^{+i w t}$ and $\mathrm{e}^{-i w t}$, the first up stimulating and the second down stimulating.

Hypothesis 2: The vacuum field is purely of the $\mathrm{e}^{-i w t}$ form, which produces the observed real component of $E$ \& $M$ fields, without having any ability to up stimulate (excite).

Hypothesis 3: All "spontaneous" emission is stimulated emission caused by the vacuum field.

While this research in the vacuum field was done for independent reasons, this result is important for a local theory of light, where nothing can be "spontaneous". In a local theory of light, everything must have a deterministic physical cause. The vacuum field is the only ubiquitous random source in the universe and an excellent candidate for producing the randomness we observe in other physical processes. For instance, this same vacuum field will provide a local solution to reflection at a dielectric interface later in this paper.

\section{Other Photonic Clues}

Over the last hundred years we have accumulated thousands of experimental clues to the true nature of light. We augment the first two clues above with a few of these that we have found especially useful in developing a local theory of light.

Clue 3: When two detectors look at a single atomic source through a beamsplitter, no simultaneous counts are observed.

This conclusion is commonly stated as: You can't split a photon. Many scientists (including the author) have tried clever ways to split one photon in two without success. The conclusion is that the photon must be a small stable primal entity - what we might call a particle. The challenge is then to integrate the particle nature of light with the many interference phenomena we use every day.

Clue 4: In a cascade transition, the detection of the first transition initiates an exponential decay of the counts from the second transition [8].

Clue 4 from Figure 3 of a 1974 paper by Clauser [8] has not been given much attention, but it provides immediate and useful insight into the photon - the part of the E \& M emission that causes excitation. He set up a cascade transition in Calcium using nanosecond detectors to measure the emission time of each sequential photon. Then he plotted the time of detection of the second photon relative to the time of detection of the first photon. He reported an exponentially decaying probability of detection for the second photon beginning exactly at the moment of detection of the first photon. Since the emission of the second photon begins with full force as soon as the first photon is emitted, we see that the photon emission comes at the time of the state transition. This experimental result is so useful that we make its conclusion the $4^{\text {th }}$ hypothesis of the local theory and list it formally:

Hypothesis 4: The photon is quite distinct and separable from the exponentially decaying $\mathrm{E} \& \mathrm{M}$ wave that precedes it. It is emitted at the moment when one emitting state transitions to the next in the cascade. Thus it appears to be at the end of the $E$ \& $M$ emission from an excited state.

On top of these rather specific constraints, we will naturally add:

Clue 5: Relativistic invariance (invariance under the usual special relativity Lorentz transformations).

Whatever physical processes we hypothesize, they should be relativistically invariant.

Clue 6: Conservation of energy, momentum and angular momentum.

The goal here is to develop a testable, deterministic, local theory which agrees with all guidelines and contains no probability waves or other nonlocal behavior.

\section{Photons as Particles in the E \& M River}

We begin the derivation of a local theory with a discussion of conservation of energy. In quantum mechanics, the energy of the photon can spread out over vast distances (even cosmic distances for galactic light), and then suddenly compress down to one location when exciting an electronic state. Where the photonic energy was until that detection and how it contributed to the gravitational field (as all energy and mass will do) and why there 
was not a small gravitational shock wave when it suddenly concentrated-these are not addressed in quantum mechanics. In contrast, a local theory must satisfy energy conservation locally where the excitation occurs.

However, the amount of classical electromagnetic field energy (measured by $|E|^{2}+|B|^{2}$ ) in the vicinity of an excited atom is in general negligible compared to the energy $h v$ of the excitation. (A $10 \mathrm{~cm}$ diameter beam carrying a single photon with a 1 um interaction area has $10^{-10}$ of the beam power in the interaction area.) So in a local theory, where could that energy come from? Even more pressing, one photonic emission creates at most one photonic detection even though the electromagnetic radiation is verified by many interferometric experiments to go over a vast space compared to an atomic size.

This discussion provides our next hypothesis.

\section{Hypothesis 5: The electromagnetic wave does not contain the excitation energy.}

While the $\mathrm{E} \& \mathrm{M}$ wave cannot contain the excitation energy, we know that the properties of the $\mathrm{E} \& \mathrm{M}$ wave and its interference with itself do affect the interaction of the photon. The polarization state and the interference envelopes of the carrier waves are routinely used to control and direct the interaction of the photon with matter, including making photons take a right turn at a beamsplitter or mirror. Thus we propose the following hypothesis for any local theory:

\section{Hypothesis 6: The familiar electromagnetic wave form is simply a carrier wave for the photon.}

This type of discussion is what led earlier scientists to create a quantum mechanical model where the squared complex field amplitude created a probability density function for the interaction. In a local theory we do not have that option, and so we must propose that the excitation part of an emission is carried along by the emitted waveform like a cork in a river. The flow of the river (the classical E \& M wave) may cover a wide expanse, but the cork (the photon) is always somewhere. In this model the cork (photon) is the trigger for an excitation (up transition) and equivalently for stimulated emission (down transition), which has an equal cross-section according to Einstein's 1917 paper cited above [7].

Hypothesis 6 and the photonic flux equation below provide the key linkage between the local theory of light and the quantum theory of light. In QM, the E \& M wave acts like a probability wave, spreading out, diffracting, splitting and recombining. In QM, the final mean-square intensity is proportional to the probability of detection. In the local theory, this $\mathrm{E} \& \mathrm{M}$ wave acts like a river carrying the photon particle, and the two theories provide the same predictions under most situations. An experiment which can potentially distinguish the local model from the QM model of light is presented in Sec 7 below.

\section{Covariant Formula for the Photonic Flux}

Clearly the river analogy requires a covariant field equation that makes the photon density match the flow of the E \& M carrier wave power. Even very complex patterns such as right angle interference of two beams, which create very high spatial frequency variations in E \& $\mathrm{M}$ power as well as right angle turns in photon direction, must have a flow pattern for the photons that matches these patterns. We show now that the Poynting Vector can be modified to provide a covariant current density for the photons as a function of wavevector $k_{\mu}$.

We need a flow rate for photonic energy in each frequency range divided by the energy of a single photon. The natural photonic flow equation would be to use the relativistic stress-energy-momentum tensor, $T^{0 v}$, as a function of frequency, since it follows the flow of energy density even in the most complex waveform interactions, and it satisfies the conservation equation in Equation (5).

$$
T^{0 \mu}{ }_{\| \mu}=0
$$

The problem here is that $T^{0 \mu}$ is not a covariant vector, but simply a piece of a tensor. We need a modification that is a true 4-vector.

To find that modification, we write out explicitly what the stress-energy-momentum tensor looks like for a plane wave. The general form of the electromagnetic stress-energy-momentum tensor is given in Equation (6a), which has the familiar conservation equation in the absence of emitting and absorbing current densities given by $6 \mathrm{~b}$ [9]. Thus Equations (6a) and (6b) apply to radiating light waves.

$$
\begin{gathered}
T^{\mu \nu}=F^{\mu}{ }_{\tau} F^{\tau v}-\frac{1}{4} g^{\mu v} F^{\tau \sigma} F_{\tau \sigma} \\
T^{\mu \nu}{ }_{\| v}=0
\end{gathered}
$$


where $F_{\mu \nu}$ is the general electromagnetic tensor given in terms of the electric and magnetic fields in Equation (7) [9].

$$
F_{\mu \tau}=A_{\mu \mid \tau}-A_{\tau \mid \mu}
$$

Substituting Equation (7) into Equation (6a), we get Equation (8).

$$
T^{\mu \nu}=\left(A^{\mu \mid \tau}-A^{\tau \mid \mu}\right)\left(A_{\tau}^{\sigma}-A_{\tau}^{\sigma}\right)-\frac{1}{4} g^{\mu \nu}\left(A^{\tau \mid \sigma}-A^{\sigma \mid \tau}\right)\left(A_{\tau \mid \sigma}-A_{\sigma \mid \tau}\right)
$$

Now use the form of $A_{\mu}=A_{\mu} \exp \left(i k^{\tau} x_{\tau}\right)+A_{\mu^{*}} \exp \left(-i k^{\tau} x_{\tau}\right)$ for a real E \& M plane wave moving through space to get the result in Equation (9), where we have simplified the result using the usual Coulomb condition $A_{\mu} k^{\mu}=0$ (which forces transverse E \& M waves) and the wave-vector condition $k^{\mu} k_{\mu}=0$, which just means the solutions are traveling at the speed of light. The very simple result is given in Equation (9), where we have dropped the $\exp \left(2 i k^{\tau} X_{\tau}\right)$ and $\exp \left(-2 i k^{\tau} X_{\tau}\right)$. Those two terms provide some local modulation of the energy density but average to zero very quickly in both space and time. For this reason they do not contribute to the mean photon flux.

$$
T^{\mu \sigma}=\left(A^{\tau} A_{\tau}^{*}\right) k^{\mu} k^{\sigma}
$$

In this case we are especially interested in the vector portion $T^{0 \sigma}$, which is the equation for the energy-momentum density.

$$
T^{0 \sigma}=\left(A^{\tau} A_{\tau}^{*}\right) k^{0} k^{\sigma}
$$

Equation (10) represents the energy-momentum density for an E \& M plane wave, but we want the photon density equation. To get that we need to divide by the energy of a single photon, which is the familiar $h v=h k^{\circ}$. The result is a covariant form of the photon current $\boldsymbol{P}^{\sigma}$ in Equation (11) for a single plane wave with wave vector $k^{\sigma}$ (which may have any density of photons in it).

$$
P^{\sigma}(k)=\left(A^{\tau}(k) A_{\tau}(k)\right) \frac{k^{\sigma}}{h}
$$

If we would like the local sum of all photon currents, we realize that $k^{\sigma}$ in Equation (12) can be gotten by differentiation. Thus the total flux of photons at each point can be written as

$$
P_{\text {tot }}{ }^{\sigma}=\frac{A^{\tau \mid \sigma} A_{\tau}^{*}}{i h}
$$

\section{Reflection from a Dielectric Surface-Critical Challenge for a Local Theory}

Let's now consider one of the hardest challenges for a local theory of light—partial reflection off a dielectric surface, as shown in Figure 1. This extraordinarily simple process forces us to identify the mechanism by which some photons (say around $4 \%$ for reflection off fused silica) will reflect while most will transmit through the surface. What is the hidden variable that determines one versus the other for each photon? Furthermore this hidden variable process must somehow scramble at each reflection so that the next reflection is equally random.

The Physical Process of Reflection: To solve the general problem of random reflection from a dielectric interface, we begin with the photon as a cork carried by the $\mathrm{E} \& \mathrm{M}$ field river, but we also require a random variable to determine which direction each photon travels when it encounters a refractive discontinuity. We have explored a random phase variable within the photon but have not found a solution that matches the usual physics and also not found a plausible way to scramble the phase after reflection. We have found a successful selection process using the vacuum field, which has a power density proportional to the omnipresent $\mathrm{h}$ as discussed above. By multiplying the intensity of each candidate path by the power in the vacuum field at the k-vector of the candidate path, we have found a process that matches conventional E \& M and QM. More specifically, each photon chooses the path (transmitted or reflected) for which the product is larger. Due to the random statistics of the vacuum field, reflection of the photons matches the power reflection of the $\mathrm{E} \& \mathrm{M}$ wave- just as in quantum mechanics.

The process consists of the following steps. 


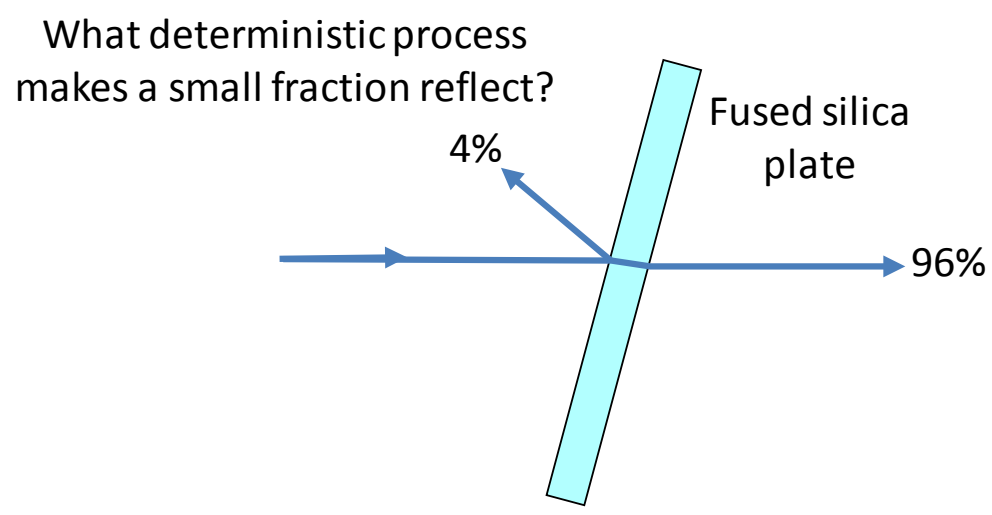

\begin{abstract}
Figure 1. The simple reflection of an E\&M wave off a dielectric interface has been a huge challenge to a local theory of light. A local theory must identify a deterministic cause for why a small fraction of the photons are reflected while most transmit. The local theory for all angles and all refractive discontinuities is presented below providing exact agreement with QM predictions.
\end{abstract}

1) When the photon comes to a refractive discontinuity, it can reflect or not.

2) To make that decision, it samples the local E \& M wave pattern, which has two components—a transmitted wave and a reflected wave.

3) Each of these two wave powers is multiplied by the vacuum field power at its particular wave vector at the location of the photon.

4) The photon then transitions to whichever path product has the larger magnitude.

This type of selection process would imply that the vacuum field may be involved in switching between candidate paths at a discontinuity.

While the detailed process of how the E \& $\mathrm{M}$ and vacuum fields are sampled is not at all defined here (and will have to wait for a field theory), the probability calculation for this process is straightforward. The probability of selecting the weaker path is equal to the probability that the power of the weaker path times the power of the vacuum field at that instant at that location with the wave-number of the path is greater than the same type of product for the stronger path. Since the local vacuum field amplitude for a particular wavevector is a Gaussian random complex number (magnitude and phase), the magnitude-squared of the amplitude (which we are using to decide which path the photon will follow) will have an exponential distribution.

$$
\begin{aligned}
& \text { Product }_{1}=\text { E\&M_Power }{ }_{1} * \text { VacuumPwr }_{1} \\
& \text { Product }_{2}=\text { E\&M_Power } 2 * \text { VacuumP } w r_{2}
\end{aligned}
$$

We will multiply each path power by the random exponential power appropriate to its wavevector, and then have the photon go in the direction of the larger product. With this process, the probability of choosing the weaker path $2\left(P_{2}\right)$ is given by the following double integral, where for conciseness we use $P w r_{1}$ and $P w r_{2}$ for the transmitted and reflected path powers, and $V a c_{1}$ and $V c_{2}$ for the vacuum field powers at each one's wavevector.

$$
P_{2}=\int_{0}^{\infty} \int_{0}^{P w r_{1} V a c_{1}<P w r_{2} V a c_{2}} \frac{\mathrm{e}^{\frac{-V a c_{1}}{\sigma_{v}}}}{\sigma_{v}} \frac{\mathrm{e}^{\frac{-V a c_{2}}{\sigma_{v}}}}{\sigma_{v}} \mathrm{dVac_{1 }} \mathrm{d} V a c_{2}=\frac{P w r_{2}}{P w r_{1}+P w r_{2}}
$$

We see that the resulting probability of the weaker path 2 being selected is exactly equal to its power compared to the total path power, as we see experimentally and as is predicted by QM.

Thus we have shown is that it is possible to construct a random selection process between two possible photonic paths that matches current $\mathrm{E} \& \mathrm{M}$ predictions and experiments. That it uses the vacuum field with its $\mathrm{h}$ factor is appealing because the randomness is completely independent at every reflection. There may, of course, be other plausible options, but all of them will require the effect of a random number generator. Even QM says that the choice is random without specifying the random process of selection. It is perhaps more difficult for QM to find a plausible random number generator since the probability wave is distributed over a large area. Having 
the interaction confined to a point in space as the photon touches the discontinuity makes it very easy to use the vacuum field-the universe's ubiquitous random field.

This process of splitting the photonic flow at any discontinuity of the E \& $\mathrm{M}$ wave is exactly what quantum mechanics does. If the $\mathrm{E} \& \mathrm{M}$ wave (interpreted as a probability wave) splits at a discontinuity, then the probability of the photon going one way or the other splits proportionately. The key conclusion about this section and its companion section 5 is that a local theory can distribute the flow of random photons identically to QM predictions even in the presence of discontinuous interfaces such as dielectric surfaces or polarizers. The rest of the time, the photon is simply following the equivalent of the power flow of the E \& M wave-what QM interprets as the probability flow. Thus the two look very close, except that the source of the randomness is clearly identified in the local theory as the vacuum field.

\section{Malus' Law-Polarized Photons through a Rotated Polarizer}

In the case of polarized light passing through a polarizer rotated by angle $\theta$ (Figure 2), we will use the same process as developed for partial reflection from a dielectric interface.

- When a photon arrives at the polarizing surface, it sees a refractive discontinuity with two E \& M carrier waves leaving that discontinuity-both of the proper frequency.

- Each path has a merit function equal to the power in its $\mathrm{E} \& \mathrm{M}$ wave times the magnitude-squared of the instantaneous vacuum field component at the wave-vector of each component.

- The photon follows whichever path has the larger product.

Just like with reflection from a dielectric surface, this process makes the probability of transmission or reflection for each photon proportionate to the relative power in each of the two E \& M fields (transmitted and reflected). Since the transmitted carrier wave has a fraction of the power equal to $\cos (\theta)^{2}$, that is the probability of transmission. This is the essence of Malus' Law and the result commonly reported by QM and experiment. This simple process assures that the photon flux matches the $\mathrm{E} \& \mathrm{M}$ wave power at each beam splitter or polarizer.

Note: To get this probability calculation to match experiment, we had to assume that the photon did its selection independent of the polarization of the two candidate carrier waves (transmitted and reflected). Otherwise whichever beam was closer to the incident polarization would have gotten a larger transmission—quite contrary to experiment. This mathematical result suggests that the photonic singularity (which we have been calling the photon) may not have a polarization associated with it at all but instead simply follows the E \& M carrier wave with whatever polarization it may have. Later experiments can test this hypothesis.

\section{Local Waveforms that Satisfy Bell's Test}

The last challenge we consider for a local theory is Bell's Theorem, which proves that two identical photons cannot combine locally to match QM predictions for count correlations through polarizers. The typical experimental layout and the verified QM prediction for count correlation are shown in Figure 3. Experimentally, scientists have worked quite hard to show that this barrier cannot be broken. Their experiments involve entangled particles (today mostly entangled photons), and they compute a count statistic that Bell's theorem says cannot be reached by a local theory. Many such experiments have been done-all agreeing with quantum predictions.

In a previous paper [2] we relaxed the definition of "entangled photons" in a manner invisible to quantum mechanics and found a local solution which bypassed Bell's Theorem and did match QM predictions. By allowing an entangled pair of photons to each has unobservable different properties, the QM predictions of count
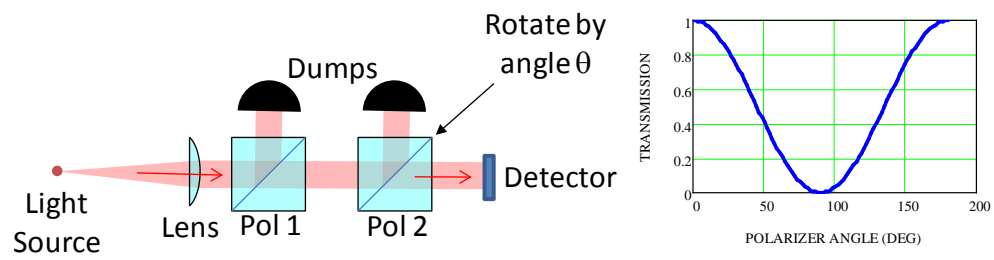

Figure 2. The simple experiment shown above passes a fraction of the incident polarized photons through a polarizer rotated by angle $\theta$. QM and experiment say that fraction is $\cos (\theta)^{2}$. A local theory must agree. 


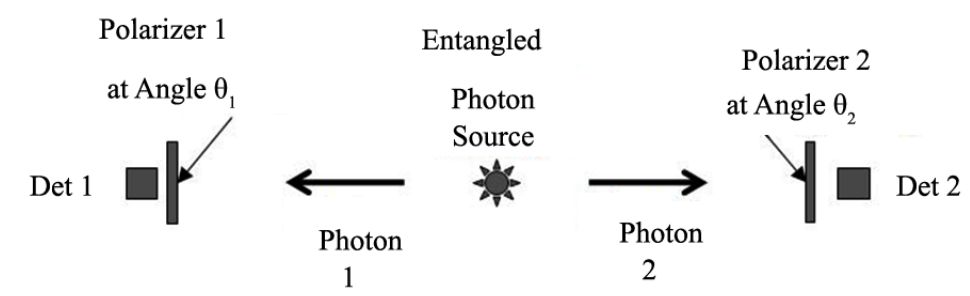

Figure 3. The Bell's Theorem test of quantum mechanics uses a source of entangled photons, where each photon goes to a different detector through two different polarizers rotated at angles $\theta_{1}$ and $\theta_{2}$. The probability of simultaneous detection is proportional to $\cos \left(\theta_{1}-\theta_{2}\right)^{2}$.

rate correlation with angle were matched, providing a local solution to Bell's Test.

To get a local theory that matched QM predictions, we hypothesized a different excitation function with polarizer angle for each of two types of photon, which together form an entangled pair. We called the excitation functions simply $\mathrm{P} 1(\theta)$ and $\mathrm{P} 2(\theta)$, where $\theta=$ the angle between the photon polarization and the polarizer axis. No one has ever found a way to measure the excitation function with angle of a single entangled photon, so these local properties we hypothesized are "unmeasurable"- true hidden variables.

We note that with this model, the two photons are still tightly entangled in that if one has excitation function $\mathrm{P} 1(\theta)$ then the other always has excitation function P2 $(\theta)$ and vice versa. In simplest terms if one photon has a detector response $\operatorname{R} 1\left(\theta_{\mathrm{ph}}-\theta 1_{\mathrm{pol}}\right)$ and the other has a detector response $\mathrm{R} 2\left(\theta_{\mathrm{ph}}-\theta 2_{\mathrm{pol}}\right)$, then if we average over all internal photon angles $\theta_{\mathrm{ph}}$, we get a correlation equal to the average of $\operatorname{R} 1\left(\theta_{\mathrm{ph}}-\theta 1_{\mathrm{pol}}\right) \operatorname{R} 2\left(\theta_{\mathrm{ph}}-\theta 2_{\mathrm{pol}}\right)$ over $\theta_{\mathrm{ph}}$, which looks like a correlation function. The result mathematically must be $\cos \left(\theta 1_{\mathrm{pol}}-\theta 2_{\mathrm{pol}}\right)^{2}$. This is, of course, the QM and experimental result.

Once we allow the two entangled photons to be different in their response to a polarizer, then we quickly find two responses with angle that together combine to match QM and experimental predictions. The two responses, however, look strange to us. One response is a step function of period $\pi$, and the other is the positive part of $\cos (2 \theta)$. As strange as they look, we note that no one has ever measured these two functions or even proposed a technique for how to measure them. Since an entangled photon comes in a random and unknown polarization state, there is no way to compare one transmission measurement with another, and thus no way to map out the angular response of a single photon. We can only do correlations as in Bell tests.

We conclude that these two functions are true hidden variables, which have never been measured and may not even be measureable. Even more, they constitute a mathematical proof that there are hidden variable formulations of light that will match Bell tests by agreeing with QM predictions.

With this two-function modification to the definition of entangled photons, we have found a solution to their count rates versus angle (plotted in Figure 4) that matched verified QM predictions. Here verification means that the correlation between the two excitation functions equals the experimental result of $\cos (\theta)^{2}$ - where $\theta=$ the angle between the two polarizer axes.

Interpretation of the curves P1 and P2: Figure 4 shows a purely mathematical result which says that if two entangled photons produce the specified count probabilities with polarizer angle, then the count rates correlate to match standard QM predictions between rotated polarizers. Now we must interpret these curves to connect to a physical model.

The blue P2 curve is the easiest to interpret. It simply says that if the polarizer axis is within $45^{\circ}$ of the photon axis, then the Type 2 photon transmits through the polarizer; otherwise it reflects.

The red P1 curve is more confusing at first. Yes the Type 1 photon transmits or reflects in the same quadrants as the Type 2 photon, but what does it mean to have more or less than one count for a single photon? The overall count average is unity over each transmissive quadrant, but what do the variations mean?

We find a clue in a previous paper [10], which showed that all photonic interactions (excitation and stimulated emission) have the same universal cross-section given by $C_{o}=\lambda^{2} / 2 \pi$, when averaged over all polarizations and directions. (This paper was based on Einstein's 1917 paper on stimulated emission [7].) Given a theory with two types of photons, we suggest that what can vary is the photonic cross-section. A value of 1 would indicate a cross-section of $C_{o}$.

If this interpretation is correct, then we have a plausible interpretation of the variable count rates in Figure 4, 


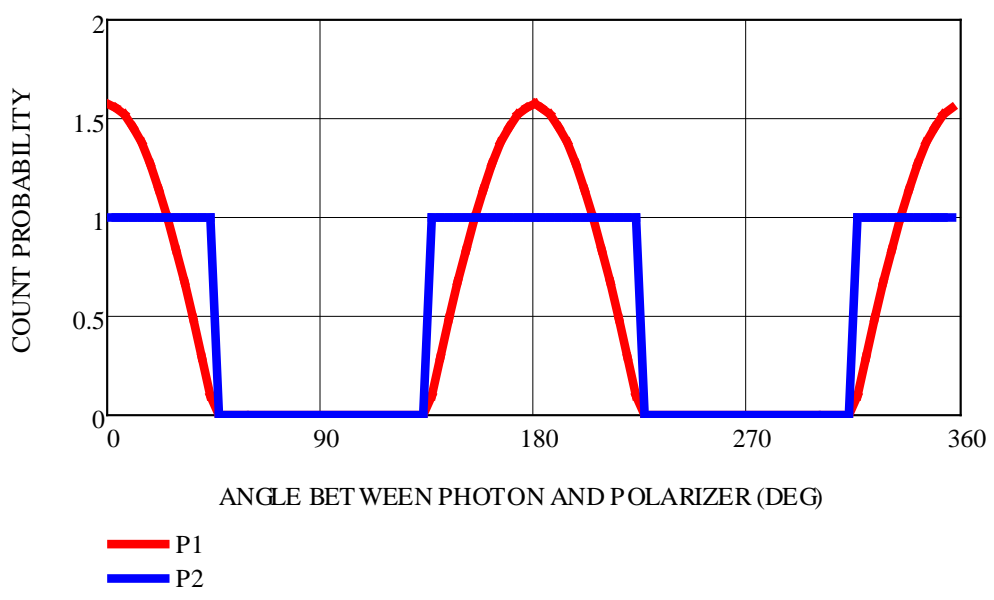

Figure 4. Plot of two photon response functions, P1 and P2, which crosscorrelate to give the experimental $\cos (\theta)^{2}$ polarization correlation predicted by QM and verified experimentally. The horizontal axis is the difference between the angle of the photon's polarization and the angle of the polarizer.

and we also have immediate physical consequences which can be verified in Bell Test experiments.

\section{High QE Test of a Local Theory vs QM}

In presenting any local theory as a competitor to the QM theory of light, it is important to identify potential experimental situations which might distinguish between the two. After all, nature will be one way or the other. Since classical E \& M theory already provides the same predictions as QM theory for many phenomena, we must go to some effort to find an experiment that will separate the local from the quantum theories. Not surprisingly, we have found that the local theory agrees perfectly with QM at low quantum efficiency, but we predict deviations from the QM predictions for count correlations with entangled photons at high quantum efficiency. That theory is presented below. It is likely that the data to test these predictions already exists, but has not been processed in the way proposed here.

To make this analysis precise, we need an equation for the probability of detection that depends on the interaction cross-section. We make this model quite simple. We assume we have a detector material of thickness $\mathrm{d}$ which has a uniform random density $n_{\text {det }}$ of detection sites/unit volume. Typically a detection site would be an atom or molecule with an electron that can be excited into a conduction band. We assume that these detection sites are distributed randomly throughout the detector material.

Using this simple model, we compute the probability of detection as follows: Each photon will follow a line path through the detector material, creating an interaction volume of its cross-section $C_{p h}$ times the thickness d. The photon's probability of being detected will then be equal to the probability that this path of volume $V=C_{p h} d$ includes a detection site.

Using the familiar Poisson statistics for a randomly distributed set of points, we compute that the probability of there being no detection is equal to the probability that there is no detection site in the interaction volume = $\exp \left(-n_{d e t} C_{p h} d\right)$. One minus that probability would then be the probability of being detected as given in Equation (16). The probability of detection is, of course, simply called the quantum efficiency of the detector, which varies with the cross-section of the photon.

$$
P_{d e t}\left(C_{p h}\right)=\left(1-\mathrm{e}^{-n_{d e t} C_{p h} d}\right)
$$

Using the definition of quantum efficiency to be the mean detection probability over all photon angles and configurations, we should get the measured $Q E$ when $C_{p h}=C_{o}$, its mean value as shown in Equation (17).

$$
Q E=\left(1-\mathrm{e}^{-n_{\text {det }} C_{0} d}\right)
$$

Now we can solve for the quantity $n_{\text {det }} d$ in Equation (16) and substitute the result into Equation (15) to get 
Equation (17) that uses only known quantities: the mean detector $Q E$ (measured by the manufacturer) and the photon interaction level divided by $C_{o}$, plotted in Figure 5.

$$
P_{\text {det }}\left(C_{p h}\right)=1-(1-Q E)^{\left(C_{p h} / C_{o}\right)}
$$

We apply this formula as shown in Figure 5 to compute the relative probability of detection for type 1 and Type 2 photons versus the angle between their polarization axis and the polarizer. That relative probability is plotted for 5 different level of quantum efficiency, $Q E$, in Figure 5. In a test of Bell's Theorem, the probability of detecting a Type 1 photon will then vary within a quadrant, while the probability of detection for a Type 2 photon will be constant in each quadrant.

Using this model for the local interaction theory, we can convolve the type 1 and type 2 curves in Figure 5, to compute the count correlation for each level of quantum efficiency, $Q E$. The deviation from QM predictions for the above local theory is quite small when the detector $Q E<0.2$ and quite substantial when the $Q E=0.99$. The comparison of local to QM predictions at $Q E=0.2$ is shown in Figure 6. When the $Q E$ of a detector is this low, this effect is almost invisible on a plot, but it can be seen numerically if the stability and count statistics of the experiment are sufficient. When the $Q E$ is 0.99 , the deviation is large and easily seen on a plot or with analysis. Figure 7 shows this case.

Local versus QM Metric: We recommend the following metric as a test for locality, using three popular measurement angles $\left(22.5^{\circ} .67 .5^{\circ}\right.$ and $\left.90^{\circ}\right)$. The ratio between the local theory and QM predictions is plotted versus Quantum Efficiency in Figure 8, showing how sensitive it is to $Q E$ getting close to unity. It is 0.707 for

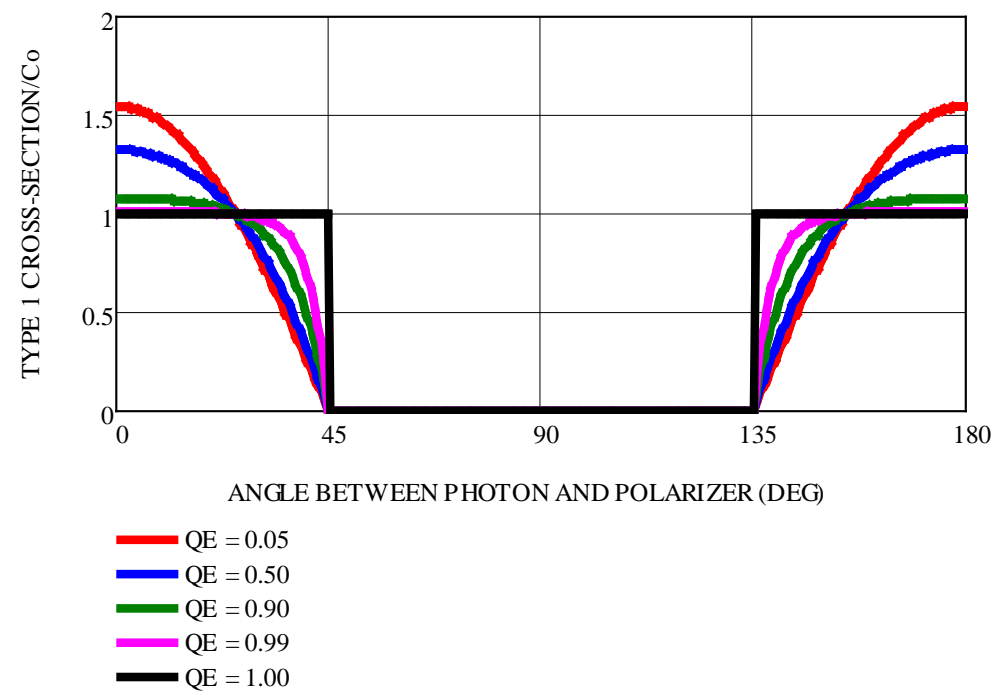

Figure 5. Here the interaction cross-section is plotted versus the angle between the Type 1 photon and the polarizer as a function of the quantum efficiency $(\mathrm{QE})$ of the detector. Since the magnitude and shape change with $\mathrm{QE}$, the local theory predicts deviations from QM predictions at high QE.
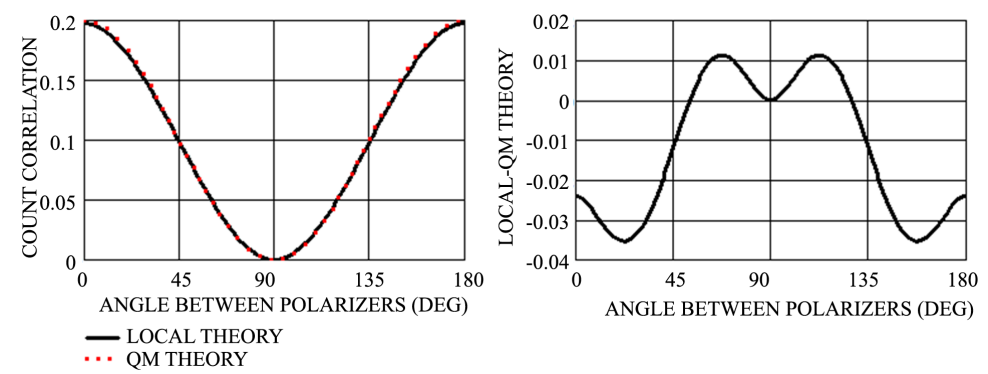

Figure 6. At detector $\mathrm{QE}=0.20$ the deviation between $\mathrm{QM}$ and local predictions is -0.0035 to +0.0011 . 


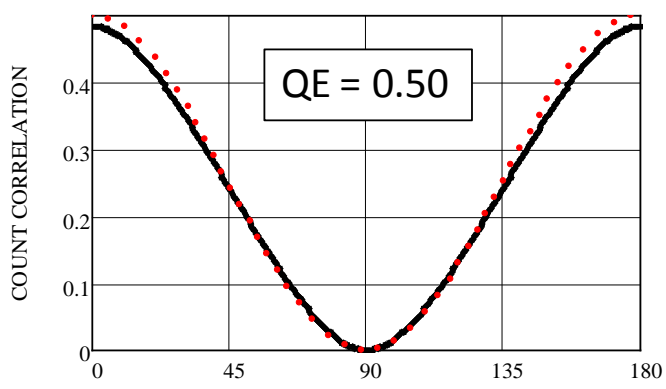

ANGLE BETWEEN POLARIZERS (DEG)

LOCAL THEORY

... QM THEORY

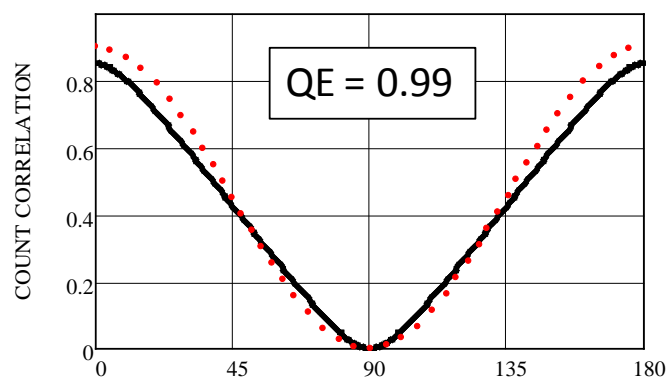

ANGLE BETWEEN POLARIZERS(DEG)

- LOCAL THEORY

-. QM THEORY

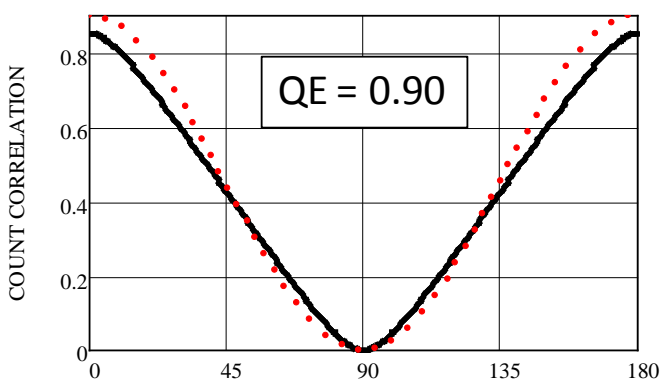

ANGLE BETWEEN POLARIZERS (DEG)

LOCAL THEORY .. QM THEORY

manc

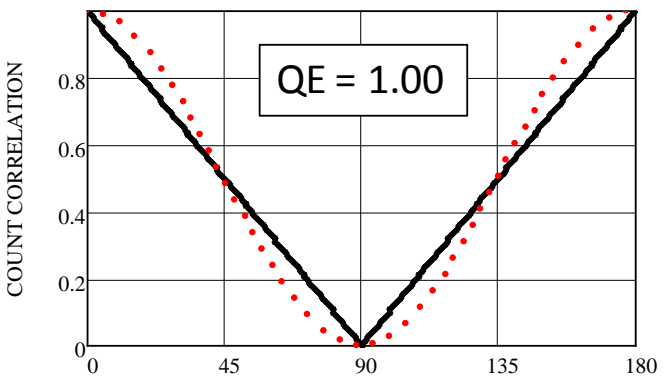

ANGLE BETWEEN POLARIZERS (DEG)

LOCAL THEORY

-. QM THEORY

Figure 7. At $Q E=0.50,0.90,0.99$ and 1.00 the deviation between $\mathrm{QM}$ and local predictions is a maximum at polarizer angles $22.5^{\circ}$ and $67.5^{\circ}$, two angles frequently in Bell tests.

$$
\text { LocalMetric }=\frac{\operatorname{Sig}(22.5 \cdot \operatorname{deg})-\operatorname{Sig}(67.5 \cdot \operatorname{deg})}{\operatorname{Sig}(22.5 \cdot \operatorname{deg})+\operatorname{Sig}(67.5 \cdot \operatorname{deg})-2 \cdot \operatorname{Sig}(90 \cdot \operatorname{deg})}
$$

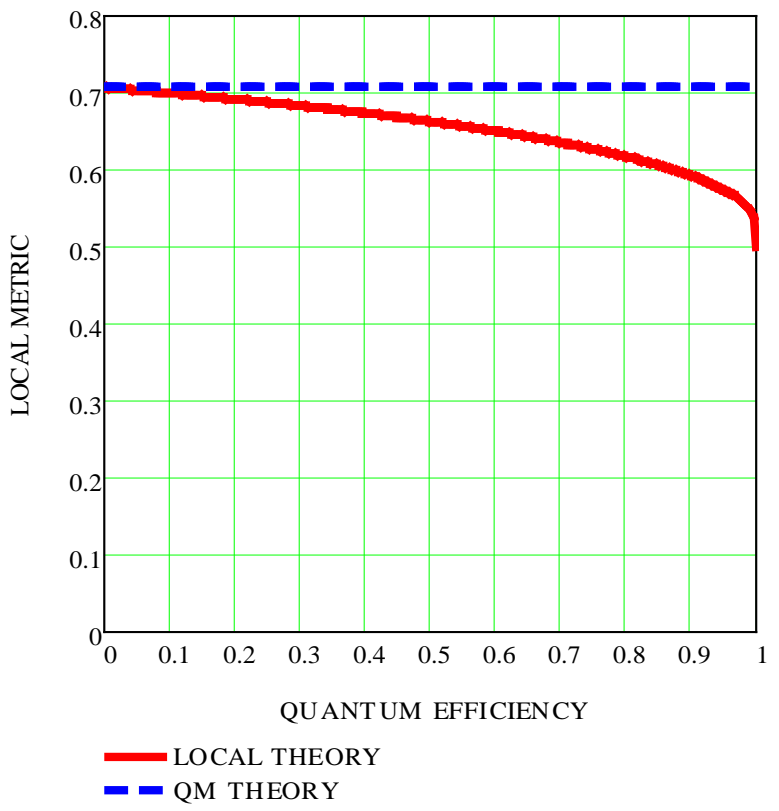

Figure 8. The Local vs QM metric is plotted here versus quantum efficiency $(Q E)$. The QE here does not include transmission loss factors along the path-just the detector. This metric is proportional to the transmission, but the fractional change between the local and QM predictions is unchanged. 
all conditions with QM models and less than that for the local theory of light.

\section{Summary}

We have explored a local theory of light by addressing four historically difficult questions:

How can a local theory of light:

1) Explain spontaneous emission of light?

2) Explain partial reflection at a dielectric interface?

3) Explain partial reflection from a polarizer (Malus' Law)?

4) Match QM results for count correlations of entangled photons?

In the first three questions we found the key in the vacuum E \& $\mathrm{M}$ field, which provided the source for spontaneous emission as well as the random number generator required for each photon to decide to reflect or not at a dielectric interface or a polarizer. QM is all about probabilistic outcomes, which require somewhere in nature a suitable random number generator. Our exploration of Questions 1, 2 and 3 suggest that the vacuum field may be central to that process - the universe's only known ubiquitous random number generator.

Question 4 (Bell's Test) was answered using a previous paper [2] on a local theory that agreed with quantum mechanics on Bell tests with entangled photons. However, in this paper we dived much more deeply to explore the physical consequences and interpretations of the local solution that emerged. The result of that deeper investigation was a correlation test for a local theory versus a QM theory of light which took advantage of the higher $Q E$ detectors available today for Bell testing.

\section{Conclusion}

The huge simplification in physics that would accompany the transition from a QM theory to a local theory compelled us (as it has others) to explore a local theory of light. Once Bell's Theorem had been bypassed mathematically [2], it seemed appropriate to explore the path to a local theory more intensely. Here we have shown possible local solutions to four historical barriers to a local field: spontaneous emission, partial reflection at a dielectric interface, partial reflection of polarized light from a polarizer, and Bell tests. An experiment which can distinguish between a local and QM model of light has been suggested. If such an experiment shows deviations from QM predictions, then the door will be opened to a local theory of light. Such experimental data may already exist in previous data sets using high $\mathrm{QE}$ detectors.

\section{References}

[1] Hutchin, R.A. (2015) A New Physical Model for the Vacuum Field Based on Einstein's Stimulated Emission Theory. Optics and Photonics Journal, 5, 109-112. http://dx.doi.org/10.4236/opj.2015.54009

[2] Hutchin, R.A. (2015) A Local Theory of Entangled Photons That Matches QM Predictions. Optics and Photonics Journal, 4, 304-308. http://dx.doi.org/10.4236/opj.2014.410030

[3] Barrow, J.D. and Tipler, F.J. (1986) The Anthropic Cosmological Principle. Oxford University Press, Oxford.

[4] Faria, A.J., Franca, H.M., Gomes, G.G., et al. (2006) The Vacuum Electromagnetic Fields and the Schrödinger Picture.

[5] Casimir, H.B.G. (1948) On the Attraction between Two Perfectly Conducting Plates. Proc. Kon. Nederland. Akad. Wetensch. B51, 793-795.

[6] Bordag, M., Mohideen, U. and Mostepanenko, V.M. (2001) New Developments in the Casimir Effect. Physics Reports, 353, 1-205. http://dx.doi.org/10.1016/S0370-1573(01)00015-1

[7] Einstein, A. (1917) Zur Quantentheorie der Strahlung (On the Quantum Theory of Radiation). Physika Zeitschrift, 18, 121-128.

[8] Clauser, J.F. (1974) Experimental Distinction between the Quantum and Classical Field-Theoretic Predictions for the Photoelectric Effect. Physical Review D, 9, 853. http://dx.doi.org/10.1103/PhysRevD.9.853

[9] Adler, R., Basin, M. and Schiffer, M. (1965) Introduction to General Relativity. McGraw-Hill, Boston.

[10] Hutchin, R.A. (2015) Universal Cross-Section of Photonic Interaction. Optics and Photonics Journal, 5, $109-112$. http://dx.doi.org/10.4236/opj.2015.54009 